\title{
Translating the power of TCM into patients' hope
}

\author{
Guangbiao Zhou $(\bowtie)^{1, a}$, Zhu Chen $(\bowtie)^{2, b}$ \\ ${ }^{1}$ State Key Laboratory of Biomembrane and Membrane Biotechnology, Institute of Zoology, Chinese Academy of Sciences, Beijing 100101, \\ China; ${ }^{2}$ State Key Laboratory of Medical Genomics and Shanghai Institute of Hematology, Ruijin Hospital Affiliated to Shanghai Jiao Tong \\ University School of Medicine, Shanghai 200025, China \\ (C) Higher Education Press and Springer-Verlag Berlin Heidelberg 2014
}

In ancient times, Chinese medical practitioners tasted medicinal herbs to test the efficacy of these herbs in treating diseases, and from the practical experience the pioneers improved their medical skills. The unique system of traditional Chinese medicine (TCM), which has evolved over thousands of years, therefore represents one of the oldest paradigms of translational medicine in the world. TCM has been widely and successfully used to treat illnesses from inflammation to cancer and retains an important role in the healthcare system of China today. However, challenges in the development of TCM have become clear in recent years, and major efforts must be made to further improve the current TCM system.

\section{The theoretical system of TCM: improvement and better interpretation are desired}

The systematic structure of the theoretical system of TCM was first described in a book published over 2000 years ago; this book was called the Inner Canon of Emperor Huang [1] and came in the form of questions and answers between Di Huang and Qi Bo. In the TCM system, humans are regarded as microcosms of the larger, surrounding universe - they interconnect with nature, are subject to its forces, and must keep a harmonious relation with it; the human body is a holistic entity of various organs that function collaboratively as five elements, and a vital energy called "qi" circulates in the body. Health is maintained by the balance between Yin and Yang, and eight principles are used to differentiate syndromes. This system, which reflects the theoretical principles and academic thoughts asso-

Correspondence: ${ }^{\mathrm{a}}$ gbzhou@ioz.ac.cn; ${ }^{\mathrm{b}} \mathrm{zchen@stn.sh.cn}$ ciated with TCM, guides practitioners in preventing and treating illnesses. However, some symptoms and phenomena cannot be explained by the eight principles, and many breakthroughs or new concepts in modern medical science have not been adapted by the theoretical framework of TCM. TCM principles are also vague to most non-TCM practitioners, regardless of their being native Chinese or foreigners. Therefore, adaptation of the essence of modern life sciences, improvement of the principles, and translation of the theoretical system of TCM into a language of modern life sciences are difficult and urgent tasks for the renascence of TCM in the new century.

\section{An unbiased attitude is needed toward TCM}

Two extreme attitudes toward TCM have emerged recently in China, and these attitudes have spurred heated discussions among practitioners and laymen. On the one hand, some argue that TCM is a pseudoscience that should be banished from public healthcare and research. On the other hand, others reckon that after thousands of years of practice and development, TCM is close to perfect and modernization will simply distort its essence [2]. In clinical practice, some physicians and patients fall into two extreme groups: one group refuses any treatment regimen containing TCM elements, whereas the other group uses only TCM therapeutics and excludes any Western medicine protocols. Loss has been reported in both groups, and an objective attitude must be encouraged to obtain maximal therapeutic efficacy for patients. Indeed, TCM and Western medicine represent two medical systems with the same goal of maintaining human health. The emerging systems medicine, which maintains the balance between the science and art of medicines with imagination and ingenuity, may provide a unique opportunity for a 
feasible convergence between TCM and western medicines while overcoming their limitations.

\section{TCM wisdom should be promoted}

The history of TCM is replete with ancient wisdoms. For example, TCM advocates that the best doctor treats the problem before it develops into a disease, and this idea represents the emergence of preventive medicine in China. TCM views the human body in a holistic manner and focuses on the patient rather than the disease. While western medicine only recently realized that treatment strategies for complex diseases should be combinatory and individualized, TCM has used these remedies for over 2000 years [1,3]. These ancient wisdoms must be promoted and introduced to the modern medical system to promote health and people (patients and the general population as a whole)oriented medical practices.

\section{Convergence of eastern and western medicine for superior therapeutics}

Balance between the science and art of medicine can be achieved by the convergence of eastern and western medicine, leading to superior therapeutics for patients. Such a paradigm may be exemplified by the development of therapeutic approaches for acute promyelocytic leukemia (APL), which was considered the most malignant type of leukemia 5.5 decades ago. According to Chinese philosophy, "educating" bad APL cells is a much better remedy than killing them. As eastern and western medicine converged, all-trans retinoic acid (ATRA) as a differentiation inducer was introduced to treat APL and complete remission rates of over $90 \%$ have been obtained [4]. The TCM principle of "taming an evil with a toxic agent" is vividly described by the action of arsenic trioxide (ATO), which targets the promyelocytic leukemia (PML)-retinoic acid receptor $\square$ (RAR $\square$ ) fusion protein [5] and significantly prolongs the life span of APL patients (particularly the relapsed ones) [6]. Moreover, the combined use of ATRA, ATO, and chemotherapy has transformed APL into a highly curable disease [7].

Formula is a therapeutic regimen containing components of "Jun" (Emperor), "Chen" (Minister), "Zuo" (Assistant), and "Shi" (Delivering Servant), all of which exert effects of mutual reinforcement and detoxification. Nearly 100000 formulas have been reported and used in TCM. Based on TCM theories for APL, the RealgarIndigo Naturalis Formula [8], in which $\mathrm{As}_{4} \mathrm{~S}_{4}$, tanshinone IIA, and indirubin represent the Jun, Chen, Zuo, and Shi elements [9], has shown powerful efficacy in clinical

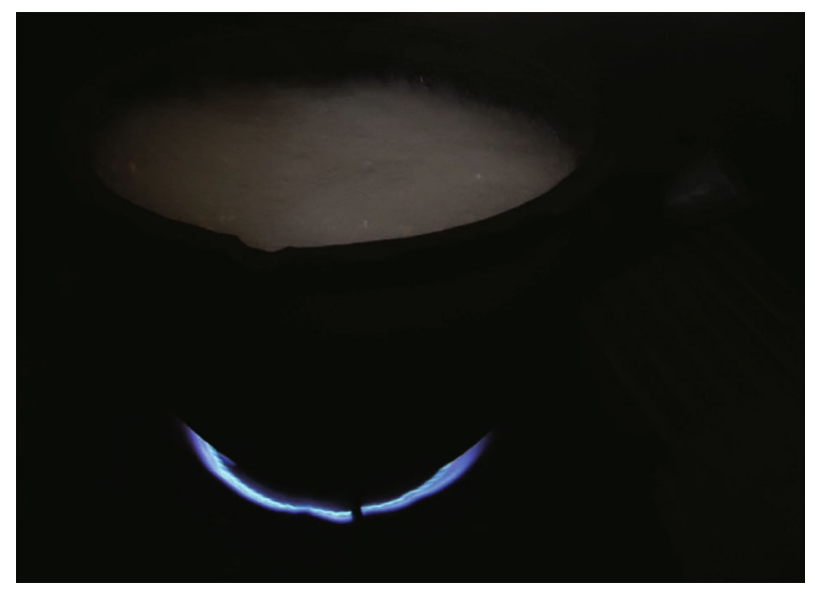

Fig. 1 Lighting the fire. With an unbiased attitude and systematic research methods, TCM will achieve its forthcoming renaissance.

trials [10]. The formula Huang Qin Tang was recently shown to be able to counteract the toxicity of chemotherapy via several mechanisms that act simultaneously [11]. Therefore, the salutary effects of TCM formulas should be explored and advocated, and rational combinatory regimens should be designed to reach maximal therapeutic efficacies for patients.

\section{Perspectives}

China is promoting both basic and clinical research to further improve TCM. The principles of TCM are apparently treasures of mankind and warrant careful investigation using systematic methods and smart strategies. Formulas that show clinical efficacy should be boosted, and their mechanisms of action, especially their effects on the immune system, intestinal flora, and the self-regulatory abilities of the body, should be dissected using systematic biomedical methods. We believe that the power of TCM can be translated into patients' hope and that the convergence of TCM and Western medicine will produce better patient outcomes than any one therapy alone.

\section{Compliance with ethics guidelines}

Guangbiao Zhou and Zhu Chen declare that they have no conflict of interest. This manuscript does not involve a research protocol requiring approval from relevant institutional review boards or ethics committees.

\section{References}

1. Original Authors Unknown. The Inner Canon of Emperor Huang (Originally published in the Spring and Autumn and the Warring 
States Periods of China, 722 BC-221 BC). Beijing: Chinese Medical Ancient Books Publishing House, 2003

2. Qiu J. China plans to modernize traditional medicine. Nature 2007; 446(7136): 590-591

3. Original Authors Unknown. Formularies for 52 kinds of disorders (Originally published in the Spring and Autumn and the Warring States Periods of China, 600 BC-400 BC). Reproduced by: Group for Management of the Silk Book in Mawangdui Han Dynasty Tomb. Beijing: Culture Relics Press, 1979

4. Huang ME, Ye YC, Chen SR, Chai JR, Lu JX, Zhoa L, Gu LJ, Wang $\mathrm{ZY}$. Use of all-trans retinoic acid in the treatment of acute promyelocytic leukemia. Blood 1988; 72(2): 567-572

5. Zhang XW, Yan XJ, Zhou ZR, Yang FF, Wu ZY, Sun HB, Liang WX, Song AX, Lallemand-Breitenbach V, Jeanne M, Zhang QY, Yang HY, Huang QH, Zhou GB, Tong JH, Zhang Y, Wu JH, Hu HY, de Thé H, Chen SJ, Chen Z. Arsenic trioxide controls the fate of the PML-RAR $\alpha$ oncoprotein by directly binding PML. Science 2010; 328(5975): 240-243

6. Sun HD, Ma L, Hu XC, Zhang TD. Ai-Lin I treated 32 cases of acute promyelocytic leukemia. Chin J Integr Chin West Med (Zhongguo Zhong Xi Yi Jie He Za Zhi) 1992; 12: 170-171 (in Chinese)

7. Hu J, Liu YF, Wu CF, Xu F, Shen ZX, Zhu YM, Li JM, Tang W,
Zhao WL, Wu W, Sun HP, Chen QS, Chen B, Zhou GB, Zelent A, Waxman S, Wang ZY, Chen SJ, Chen Z. Long-term efficacy and safety of all-trans retinoic acid/arsenic trioxide-based therapy in newly diagnosed acute promyelocytic leukemia. Proc Natl Acad Sci USA 2009; 106(9): 3342-3347

8. Huang SL, Guo AX, Xiang Y, Wang XB, Lin HX, Fu L. Clinical study on the treatment of acute promyelocytic leukemia with Composite Indigo Naturalis tablets. Chin J Hematol (Zhonghua Xue Ye Xue Za Zhi) 1995; 16: 26-28 (in Chinese)

9. Wang L, Zhou GB, Liu P, Song JH, Liang Y, Yan XJ, Xu F, Wang BS, Mao JH, Shen ZX, Chen SJ, Chen Z. Dissection of mechanisms of Chinese medicinal formula Realgar-Indigo naturalis as an effective treatment for promyelocytic leukemia. Proc Natl Acad Sci USA 2008; 105(12): 4826-4831

10. Chen SJ, Zhou GB, Zhang XW, Mao JH, de Thé H, Chen Z. From an old remedy to a magic bullet: molecular mechanisms underlying the therapeutic effects of arsenic in fighting leukemia. Blood 2011; 117(24): 6425-6437

11. Lam W, Bussom S, Guan F, Jiang Z, Zhang W, Gullen EA, Liu SH, Cheng YC. The four-herb Chinese medicine PHY906 reduces chemotherapy-induced gastrointestinal toxicity. Sci Transl Med 2010; 2(45): $45 \mathrm{ra} 59$ 